\title{
North of England Dermatological Society
}

Sitzung in Leeds vom 10. Juni 1932

(The Br. j. o. D. a. 8. 1932, 8-9.)

Ingram: 1. Ungewöhnlicher Fall von Lupus erythematodes. 35jährige

Frau mit infiltriertem, ringförmigen Erythem, das, später schuppend, linke

Wange ausfüllte. Seit kurzem so was wie Granuloma annulare mit livid schup-

Periodische Literatur.

61

pender Scheibe an Nasenspitze. Krysolgan anfangs mit Erfolg. 2. Fall von Rosakrankheit. 7monatiges Kind mit generalisiertem, morbilliformen Erythem, Lichenifikation an Genitalien und inneren Oberschenkeln. Starkes Schwitzen und Abschilferung an Hohlhänden und Fußsohlen. Photophobie und Hypertonie. 3. Epidermolysis bullosa. 10 Jahre altes Kind, das seit frühester Jugend ergriffen, jetzt Narben und Epidermalzysten an vorstehenden Stellen und Nageldystrophie zeigt. Mutter litt an gleichem Zustand. 4. Pall zur Diagnose. 70jähriger Mann seit 12 Monaten mit Hornpapeln an Handrücken und Pingern. Einzelläsion anfangs wie leicht schmerzender Fleck, der sich allmählich zu erbsengroßen hornigen Papeln auswächst mit stecknadelkopfgroßem, schwarzen, glatten, durchscheinenden Hornpflock im Zentrum. Mikroskopisch nur Hyperkeratosis. 5. Onycholysis. 14jähriger Junge verlor seit 18 Monaten seine N.ägel. Alle Nägel ergriffen. 6. Tuberkulide bei 66jährigem Manne. Eingförmige hyperkeratotische Infiltration auf linker Hüfte mit mikroskopischer tuberkulöser Struktur. Dauer 12 Monate. Vor 5 Monaten waren zahlreiche purpurfarbene hyperkeratotische Läsionen an beiden Unterschenkeln erschienen mit einer großen Hämorrhagie hinter rechtem Knie, die auf Bettruhe verschwand. Wa.R. negativ. Achlorhydrie. Keine Anzeichen für innere Tbc.Siegfried (Bad Aachen).

Periodische Literatur.

Zusammengestellt von Dr. Arthur Alexander in Charlottenburg.

Gonorrhoe.

Therapie.

Sachs, B., Ein Beitrag zur Frage der Fluorbehandlung mit Goldpräparaten.

Munch, med. Wschr. Nr. 42. 1931.

Die Dazetpräparate, organische Goldverbindungen mit sulfosauren Benzolderivaten, besitzen gegenüber dem auf infektiöser, unspezifischer Basis entstandenen Scheidenfluor ausgezeichnete desinfizierende und heilende Eigen-schaften. Sie treten sowohl bei dem flüssigen Präparat, wie in Puder- und Salbenform (Vaginalkugeln) zutage. Sie sind einer großen Zahl anderer Mittel dadurch überlegen, daß sie die desinfizierenden Eigenschaften auch in einem schleim- und eiweißhaltigen Milieu durch störende adsorptive Bindungen nicht verlieren, keine Eiweißfällungen und keine Ätzwirkungen verursachen. Eine granulationsanregende, den 
stofflichen Umsatz fördernde Komponente be-günstigt eine rasche Heilung entzündlicher Schleimhautveränderungen und von Erosionen der Portio. Die günstige Einwirkung zeigt sich auch bei dem nicht infektiösen, sogenannten essentiellen Fluor. Letzterer wird auch allein durch - die orale Gabe des anregenden Faktors, des Zetton, einer besonderen Gold-verbindung mit stark katalysierender Wirkung, günstig beeinflußt.

$\mathrm{We}^{1 / 2} / 2 \operatorname{sicoe}^{7 / 8}-$ Charlottenburg.

Reinhardt, J., Beitrag zur Fluorbehandlung. Die Medizinische Welt. Nr. 51. 1931.

Globulaktin-Kugeln, die Milchsäure in statu nascendi abscheiden, haben eine gute Wirkung bei einfachem Scheidenkatarr $1 / 8$ Globulaktin-Kugeln komp., 